\title{
Infección respiratoria por virus influenza en niños: ¿Q ué aprendimos durante el año 2004?
}

\author{
Luis E Vega-Briceño $2 a$, Marcela Potín ${ }^{1}$, Pablo Bertrand ${ }^{2}$, \\ Ignacio Sánchez². \\ Clinical features of respiratory \\ infections due to influenza virus \\ in hospitalized children
}

Background: Infants and toddlers have the highest influenza hospitalization rate in pediatrics. Although the impact of this virus in children has been recognized, there is no defined statement related to vaccination in this population. Aim: To describe clinical and epidemiological characteristics of complicated influenza infections in hospitalized children. Material and methods: All hospitalizations due to influenza virus were recorded prospectively between March and June 2004. Results: We registered 40 laboratory-confirmed influenza admissions. Median age was 24 months (range: 15 days-14.5 years), 52\% males, 18 younger than 2 years. Most of them had an underlying medical condition. The most common conditions were recurrent wheeze in 17, a neurological disease in 7 and asthma in 6 . Twenty had more than one condition and 15 were previously healthy. The average days of respiratory symptoms and fever prior to admission were 5 and 3, respectively. The most common discharge diagnoses were concomitant viral-bacterial pneumonia in 53\%, viral pneumonia in 38\% and laryngitis in $8 \%$. Influenza virus A was identified in 34/40 children. Oxygen supplementation was required by 34 cases; $20 \%$ of which required an $\mathrm{O}_{2}$ inspired fraction over $40 \%$. The average days of hospitalization and oxygen were 4 and 3, respectively. Eleven children were treated with amantadine and 21 with antimicrobials. Four children were admitted to pediatric intensive care units and two cases required non-invasive ventilatory support. No deaths were recorded. Conclusions: Our data confirms the importance of influenza virus infection in children, as measured by admission rates, complications and length of hospital stay. Young children are a risk group for which immunization is recognized as protective (Rev Méd Chile 2005; 133: 911-18).

(Key Words: Age groups, child; Influenza virus A; Orthomyoxoviridae; Pneumonia)

\footnotetext{
Recibido el 8 de noviembre, 2004. Aceptado el 19 de abril, 2005.

${ }^{1}$ Servicio de Pediatría y ${ }^{2}$ Sección Respiratorio Pediátrico, Departamento de Pediatría, Pontificia Universidad Católica de Chile. Santiago de Chile.

aBecado del Programa Enfermedades Respiratorias del Niño, Departamento de Pediatría, Pontificia Universidad Católica de Chile.
}

Correspondencia a: Dr. Luis Enrique Vega-Briceño. Lira 85, 5o piso, Santiago Centro. Casilla 114-D. Laboratorio Respiratorio Pediátrico, Departamento de Pediatría, Pontificia Universidad Católica de Chile. Teléfono: 354-3767. Fax: 247-3879. E mail: levega@puc.cl 
$\mathrm{E}^{1}$ impacto clínico y epidemiológico de la infección por virus influenza (VI) en niños es significativo. Cerca de $20 \%$ de ellos, a nivel mundial, desarrolla síntomas respiratorios cada año, siendo los más pequeños el grupo que concentra el mayor número de hospitalizaciones en la edad pediátrica ${ }^{1-3}$. Las manifestaciones clínicas del VI varían desde los síndromes respiratorios con odinofagia, coriza, tos y fiebre alta, hasta la neumonía viral o sus complicaciones por sobreinfección bacteriana (otitis, sinusitis o neumonía) ${ }^{4,5}$. La evolución clínica depende de diversos factores, como la edad, estado inmunitario, condiciones cardiorrespiratorias crónicas de base y la virulencia de la cepa circulante ${ }^{6-8}$.

La vacunación anual contra el VI se ha recomendado históricamente para los individuos mayores de 65 años, por constituir éste un grupo de riesgo ${ }^{9,10}$. Recientemente, se incorporó a esta recomendación, a todos los niños entre 6 y 23 meses, en especial aquellos con alguna condición crónica de base ${ }^{10,11}$. Por otro lado, se reconoce que los niños menores de 5 años, incluso sin condiciones de riesgo conocidas, presentan tasas de hospitalización y complicaciones similares a los adultos mayores de 65 años ${ }^{2,12,13}$. A pesar de ello, el uso de la vacuna contra VI en niños es aún muy limitado, probablemente por una percepción inexacta que la infección por el VI es una enfermedad benigna en niños ${ }^{13,14}$.

El impacto de la infección por VI debe ser medido empleando indicadores epidemiológicos correctos. Es probable que el número de hospitalizaciones, uso de antibióticos o el incremento del número de consultas externas sean los más apropiados $^{2}$. La tasa de mortalidad en niños no es un buen criterio para evaluar el impacto de esta epidemia ${ }^{2,15}$. Durante el reciente brote epidémico del VI en el hemisferio norte, se observó una aparición más precoz de los casos, así como un incremento en la gravedad de los niños hospitalizados $^{16,17}$. Esto motivó el desarrollo de este estudio, con el objetivo de describir las características clínico-epidemiológicas de la infección por VI complicada en los niños hospitalizados en el Servicio de Pediatría del Hospital Clínico de la Universidad Católica (UC) durante el brote del año 2004.

\section{Pacientes y MÉTodo}

De manera prospectiva, se registraron todas las hospitalizaciones de niños menores de 15 años asociadas a VI en el Hospital UC entre marzo y junio de 2004. Todos los niños ingresaron a través del Servicio de Urgencia, siendo incluidos aquellos con confirmación virológica de infección mediante técnicas de inmunofluorescencia o Test pack ${ }^{\circledR}$. Se aplicó un cuestionario estandarizado a la madre, preguntando por condiciones crónicas de base, factores de riesgo, síntomas y signos respiratorios.

Durante las primeras $12 \mathrm{~h}$ de su ingreso, se practicó un hisopado nasofaríngeo a todos los niños con sospecha de infección por VI; en breve, la muestra se homogenizó y centrifugó (10 min a $3.500 \mathrm{rpm}) ;$ se aplicó la muestra sobre dos placas (influenza A y B, respectivamente), se dejó secar a temperatura ambiente y luego se fijó con acetona. Se tiñó con el reactivo DFA (PathoDx, DPC Los Angeles, CA 90045) y se incubó a $37^{\circ} \mathrm{C}$ en una cámara húmeda; se lavó dos veces con PBS y se secó; se agregó una muestra de glicerina tamponada para ser observada en el microscopio de inmunofluorescencia.

Todos los niños con VI confirmado fueron evaluados diariamente por un mismo médico, quien registró las características clínicas y epidemiológicas. Se registraron los exámenes de laboratorio y radiológicos solicitados por los médicos tratantes, así como las razones de cada solicitud y el cambio en el manejo de cada paciente luego de conocido el resultado. Finalmente, se registraron los tratamientos antibióticos y antivirales indicados, incluyendo dosis y número de días de administración.

Para efectos de este estudio, se consideró como probable neumonía bacteriana a los niños con fiebre y crepitaciones pulmonares que tuvieron cambios en el hemograma, asociado a un ascenso en la PCR de al menos dos veces el valor de ingreso; hipoxemia a la saturación arterial de oxígeno menor o igual a $90 \%$ por al menos 2 min; condición de base a la presencia de prematurez, alergia, enfermedad neurológica, genopatía; factor de riesgo a la presencia de alguna condición que incremente el riesgo de complicaciones derivadas de la infección por VI tales como patología cardiorrespiratoria crónica, inmunodeficiencias 0 
malignidades hematológicas ${ }^{11}$; sibilancias recurrentes como la presencia de $\geq 3$ episodios de sibilancias confirmadas por médico y niño sano a aquellos sin ninguna de las condiciones antes mencionadas. Se utilizó como criterio de alta: temperatura axilar $<37,5^{\circ} \mathrm{C}$ (al menos $24 \mathrm{~h}$ ), buena tolerancia oral $(100 \%$ de su requerimiento) y ausencia de hipoxemia al menos $24 \mathrm{~h}$. En todos los casos de realizó un control telefónico a los 15 días posteriores al alta, mediante una entrevista estandarizada preguntando por eventuales nuevas consultas o síntomas asociados. En este estudio no se reportan los hallazgos radiológicos, que serán materia de otra publicación.

Estadística. Se empleó el paquete SPSS para Windows; considerando todas la variables continuas o categóricas, siendo las variables numéricas expresadas en números enteros. Para las primeras, se empleó la prueba de t de Student o MannWhitney (para aquellas variables que no cumplían criterio de normalidad) y Chi cuadrado para las segundas, considerando diferencias estadísticamente significativas aquellas con $\mathrm{p}<0,05$.

\section{RESULTADOS}

Características y diagnóstico. Durante el período de estudio se registraron 224 hospitalizaciones por causas respiratorias en el Servicio de Pediatría de la UC, de las cuales 40 (18\%) correspondieron a complicaciones del VI. La Figura 1 muestra la distribución del VI durante el reciente brote 2004, observando que $90 \%$ de las hospitalizaciones se concentraron entre mayo y junio. La edad promedio de los niños fue 24 meses (rango 15 días-14,5 años) siendo 9/40 menor de 1 año, 18/40 menor de 2 años y 53\% varones. El promedio de días de hospitalización fue de 4 (rango: 1-14 días). El 63\% de los pacientes requirió hospitalización por al menos 4 días. La Tabla 1 muestra las características clínicas de los niños hospitalizados. Veinte niños tenían al menos dos condiciones de base, siendo $92 \%$ portadores de asma o sibilancias recurrentes. En 21/25 se identificó al menos un factor de riesgo para evolución grave de la infección por VI. Sólo 5/40 habían recibido inmunización contra VI ese año. Los niños menores de 2 años con alguna condición médica de base



Figura 1. Distribución del número total de pacientes con influenza en relación al número total de infecciones respiratorias durante el período marzo-junio 2004. 


\section{Tabla 1. Características dínicas de 40 niños hospitalizados por influenza durante el período marzo-junio 2004}

\begin{tabular}{|lrr|}
\hline & N & $\%$ \\
\hline Sano previamente & 15 & 37 \\
Condición de base & 25 & 63 \\
$\quad$ Sibilancias recurrentes & 17 & 68 \\
Enfermedad neurológica & 7 & 28 \\
Asma & 6 & 24 \\
Prematurez & 6 & 24 \\
Alergia/Atopia & 5 & 20 \\
Cardiopatía & 3 & 12 \\
Síndrome de Down & 3 & 12 \\
Inmunodeficiencia & 2 & 8 \\
Ventilación domiciliaria & 2 & 8 \\
Otros & & \\
Asistencia sala cuna & 11 & 44 \\
Uso de antibiótico reciente & 13 & 32 \\
Consulta previa reciente & 27 & 68 \\
\hline
\end{tabular}

tuvieron en promedio 1 día más de hospitalización. Se solicitaron un total de 12 Test pack ${ }^{\circledR}$ y 33 paneles respiratorios, identificando al virus influenza A en 34/40 casos (85\%); 6 por Test pack ${ }^{\circledR}$ y 28 por IFD. Cinco Test pack ${ }^{\circledR}$ fueron negativos, aunque el panel viral fue posteriormente positivo para VI tipo A. Se encontró coinfección con otros virus respiratorios en sólo dos casos (parainfluenza 2 y VRS).

Hallazgos clínicos. La Tabla 2 muestra los signos y síntomas de los niños hospitalizados por VI. La mayoría de niños consultaron por tos y fiebre, presentando más de la mitad de ellos dificultad respiratoria. Se registró hipoxemia en 34/40 niños al momento de ser admitidos a sala. El promedio de días de oxígeno fue de 3 (rango: 1-12 días). Los niños con VI A y B tenían en promedio 3,5 y 2 días de fiebre, respectivamente al momento de su ingreso $(p=0,16)$. Los niños con VI tipo A mostraron mayor compromiso del estado general. Dos niños presentaron convulsión febril asociada al VI. Se registraron además otros síntomas como vómitos $(28 \%)$, dolor abdominal (28\%) o cefalea 0 dolor torácico (evaluado en los mayores de 3
Tabla 2. H allazgos clínicos en $\mathbf{4 0}$ niños hospitalizados por influenza durante el período marzo-junio 2004

\begin{tabular}{|lrr|}
\hline Síntoma/signo & $\mathrm{n}$ & $\%$ \\
\hline Fiebre & 39 & 98 \\
Tos & 37 & 93 \\
Hipoxemia & 34 & 85 \\
Retracciones & 34 & 85 \\
Crepitaciones & 31 & 78 \\
Dificultad respiratoria & 28 & 70 \\
Sibilancias & 27 & 68 \\
Rinorrea & 26 & 65 \\
Decaimiento & 23 & 58 \\
Compromiso del estado general & 15 & 38 \\
Vómitos & 11 & 28 \\
Desnutrición & 5 & 13 \\
Irritabilidad & 5 & 13 \\
Cianosis & 4 & 10 \\
Estridor & 3 & 8 \\
Diarrea & 3 & 8 \\
\hline
\end{tabular}

años) en $20 \%$. No se registraron mialgias. En 34/40 niños se presentó dificultad para la alimentación requiriendo fluidos endovenosos.

El número promedio de días de síntomas respiratorios y fiebre antes de la hospitalización fue 5 (rango: 2-17 días) y 3 (rango: 1-14 días), respectivamente. El $20 \%$ de los niños requirieron $\mathrm{FiO}_{2}>40 \%$; este grupo tuvo una estadía hospitalaria y requerimientos de oxígeno más prolongados que sus controles $(p<0,05)$. Los niños con $\mathrm{FiO}_{2}$ $>40 \%$ emplearon antibióticos 1,5 veces más que sus respectivos controles. Los diagnósticos de egreso más frecuentes fueron: neumonía con sobreinfección bacteriana $(52,5 \%)$, neumonía viral $(37,5 \%)$, laringitis obstructiva (8,4\%). En 3 niños se consignó, además, otitis media aguda (OMA). Durante los meses de mayo y junio se registraron 20 y 26 hospitalizaciones por neumonía, respectivamente, siendo el VI responsable de $95 \%$ en mayo y $50 \%$ en junio (Figura 2). Finalmente, cuatro niños ingresaron a la Unidad de Cuidados Intensivos (1 era previamente sano), requiriéndose soporte ventilatorio no invasivo en dos casos; la edad promedio fue de 4,5 años, con un promedio de hospitalización y fiebre de 8,5 y 6,5 


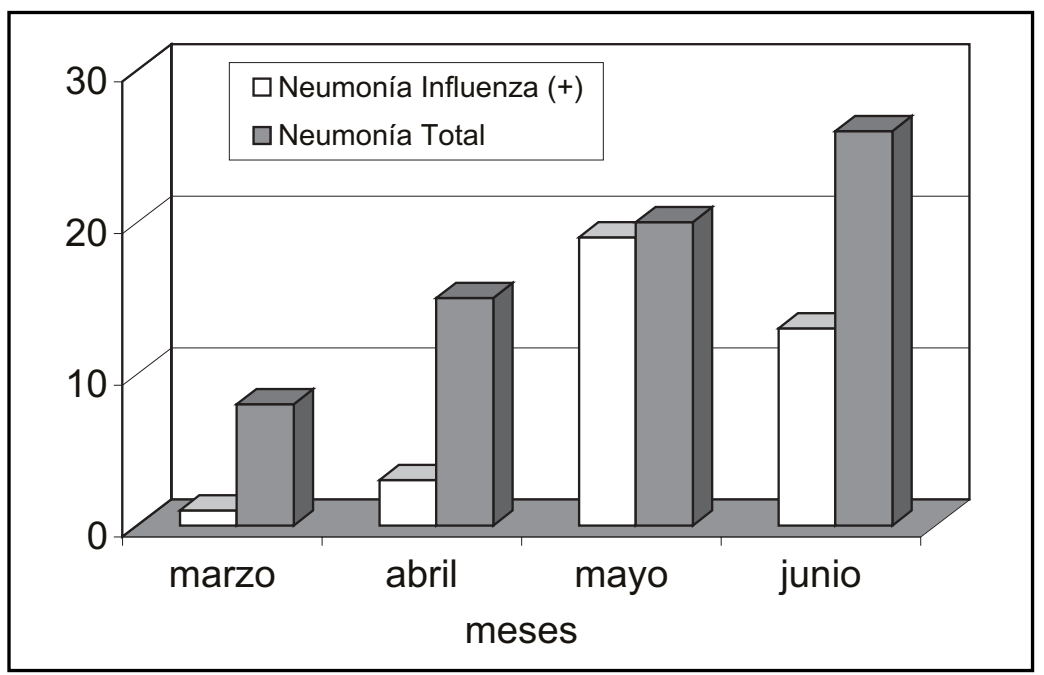

Figura 2. Distribución del número de pacientes con neumonía por influenza en relación al número total de neumonías hospitalizadas según meses durante el período marzo-junio 2004.

días, respectivamente. Todos presentaron neumonía con sobreinfección bacteriana de acuerdo con nuestros criterios. No se registraron muertes en esta serie.

Hallazgos de laboratorio. Treinta y cuatro niños tenían hemograma al ingreso; 22 de ellos tenían recuento de leucocitos normales en ese momento, 2 mostraron leucopenia $\left(<4.000\right.$ células $/ \mathrm{mm}^{3}$ ) y 10 leucocitosis $\left(>15.000\right.$ células $\left./ \mathrm{mm}^{3}\right)$. Durante las primeras $48 \mathrm{~h}$ ocurrieron cambios en el hemograma, con desviación a izquierda en 11 casos. Este viraje del hemograma, asociado a un aumento en los requerimientos de oxígeno, cambios en la signología pulmonar o deterioro en el aspecto general del niño, motivó el inicio de antibióticos en todos los casos. La PCR al ingreso (VN: $<1 \mathrm{mg} / \mathrm{dl}$ ) se solicitó en 38 niños, estando alterada en 34/38. Veintiún niños experimentaron un incremento de la PCR al menos dos veces sobre su valor basal. Se solicitaron hemocultivos en 10 pacientes, obteniendo algún germen en sólo 1 de ellos (S coagulasa negativo).

Tratamiento. En esta serie se emplearon antibióticos en 21 pacientes, por las siguientes indicaciones: sospecha de neumonía sobreinfectada bacteriana (20) u OMA (3), siendo los más frecuentes penicili- na o amoxicilina (17) y cefotaxima asociado a cloxacilina (4). Se usó amantadina en 11 pacientes; en 7 casos asociada a antibióticos.

\section{Discusión}

Este estudio prospectivo reveló la presencia del VI en $18 \%$ de los niños hospitalizados por alguna condición respiratoria durante el brote epidémico del año 2004; casi la mitad de ellos fueron menores de 2 años, con alguna condición de base como asma o sibilancias recurrentes en forma muy similar a lo reportado en la literatura 2,12,18,19. El 88\% de nuestra serie no había recibido vacuna antiinfluenza, cifra similar a lo comunicado en países industrializados ${ }^{20,21}$. Es probable que muchas de estas infecciones y hospitalizaciones graves pudieron haber sido prevenidas con una inmunización adecuada ${ }^{22}$.

Durante el brote de influenza de 2004, la tasa estimada de infección por VI que se registró en Chile fue 824/100.000 habitantes; 2 a 3 veces superior a los años previos ${ }^{15}$, lo que se tradujo en un incremento significativo en el número de consultas respiratorias ambulatorias y hospitalizaciones. Lograr estimar la verdadera incidencia del VI en niños es difícil ya que muchos de ellos tienen 
escasos síntomas y no consultan; sin embargo son una importante fuente de propagación de la infección a niños, adultos e individuos de alto riesgo ${ }^{8}$. En nuestro centro se registró un aumento de atenciones respiratorias durante los meses de invierno, con un comienzo más precoz que en otros años. Delpiano y cols publicaron recientemente su experiencia con la epidemia del año 199923. Ellos reportaron $51 \%$ de complicaciones por VI en una serie de pacientes significativamente más pequeños que los nuestros (60\% eran menores de 12 meses). $\mathrm{Al}$ igual que en esa temporada, la máxima actividad de VI durante 2004 se registró en mayo (alrededor de la semana 21).

El cuadro clínico de la infección por VI varía según la edad ${ }^{24}$. En los niños más pequeños es difícil distinguir el cuadro febril del VI de los otros síndromes febriles del lactante. En este estudio los síntomas y signos más frecuentes como fiebre, tos y dificultad respiratoria, no ayudaron a diferenciar este cuadro febril de otras infecciones virales. Los síntomas gastrointestinales, cefalea y mialgias, fueron infrecuentes en esta serie. El VI típicamente se presenta por cuadro de fiebre brusca y alta, pudiendo producir incluso convulsión febril, como en 2 pacientes de nuestra serie. Es destacable que $10 \%$ de los niños hospitalizados requirió manejo en la UCI y 2 de ellos soporte ventilatorio no invasivo. En niños, la infección por VI puede producir cuadros muy graves y eventualmente la muerte $^{5}$.

La presentación inespecífica de esta infección hace necesario una rápida y efectiva herramienta de laboratorio. La infección por VI fue identificada mediante el estudio de paneles virales en $83 \%$ de los casos. Si bien el VI es responsable de hasta $30 \%$ de los cursos de antibióticos administrados durante la temporada de influenza, el diagnóstico precoz de laboratorio podría disminuir el uso de antibióticos innecesarios ${ }^{2,25}$. La OMA fue encontrada en 3/40 niños, lo cual es significativamente menor que lo reportado en la literatura ${ }^{26}$. Se reconoce que la infección por VI predispone el desarrollo de $\mathrm{OMA}^{27}$. La vacuna ha demostrado una reducción significativa en la incidencia de OMA asociada a la infección por $\mathrm{VI}^{11}$.

El 90\% de los niños presentó neumonía asociada a VI; 15 casos fueron virales y 21 posibles sobreinfecciones bacterianas, lo que representó hasta $95 \%$ de las etiologías de neumonías durante mayo. Juven y cols encontraron $4 \%$ de VI en 254 niños con neumonía de la comunidad durante un período de un año. Demostraron además infección mixta entre influenza y Streptococcus pneumoniae en la mitad de los pacientes ${ }^{28}$. El antecedente de infección por VI está asociado con la presencia de neumonía grave por neumococo en niños ${ }^{29}$. Las características del hemograma y la PCR puede ayudar a distinguir una posible sobreinfección bacteriana.

$\mathrm{Si}$ bien la literatura muestra que los niños pequeños con alguna condición de base tienen hospitalizaciones más prolongadas al presentar complicaciones de influenza ${ }^{30}$, en este estudio se observó esta tendencia pero no alcanzó significación estadística, probablemente por el tamaño muestral reducido y la dispersión misma de los datos. Algunas limitaciones de este estudio incluyen el posible subregistro de casos. El primer paciente con VI confirmado se identificó el 30 de marzo y si bien durante ese mes no se realizó una pesquisa rutinaria de virus respiratorios, es poco probable que el número de casos de VI (durante marzo) fuese significativo. Con relación a la muestra de secreción nasofaríngea, se obtuvo una muestra por niño mediante técnica estandarizada y por personal de enfermería entrenado. La calidad de la muestra fue evaluada a través de su celularidad, siendo repetidas aquellas inadecuadas. El procesamiento y lectura de la IFD la realizó una misma persona en el laboratorio. Aunque este estudio no proporciona las bases para evaluar el costo beneficio de la vacuna, encontramos un incremento significativo en el número de hospitalizaciones y neumonías asociadas a VI. Es probable que muchos niños con infección no consulten un Servicio de Urgencia ${ }^{22}$, sin embargo aquellos que sí lo hacen y se hospitalizan experimentan un elevado número de días de estadía hospitalaria con requerimientos de oxígeno en la mayoría. Debe de considerarse el comportamiento epidémico del VI, el cual muestra importantes variaciones clínicas según el año y la cepa circulante; por lo que nuestra experiencia no puede ser extrapolada a otros años.

En resumen, esta serie mostró el impacto del VI en términos de morbilidad durante la temporada de invierno 2004. Muchos de estos episodios potencialmente pudieron ser prevenibles, especialmente por el alto número de niños con 
condiciones de base. Los cálculos epidemiológicos sugieren que incluso en los niños sanos, la vacunación es costo-efectiva ${ }^{11,31}$. Se estima que la vacunación reduciría la tasa de complicaciones por VI y muchas de estas hospitalizaciones serían

\section{REFERENCIAS}

1. Turner D, Wailoo A, Nicholson K, Cooper N, Sutton A, Abrams K. Systemic review and economic decision modeling for the prevention and treatment of influenza A and B. http:// www.nice.org.uk/pdf/influenzaassrep.pdf (Revisado el 30 marzo de 2004).

2. Neuzil KM, Mejen BG, Wright PF, Mitchel EF JR, GrifFin MR. The effect of influenza on hospitalizations, outpatient visits, and courses of antibiotics in children. N Engl J Med 2000; 342: 225-31.

3. Long CE, Hall CB, Cunningham CK, Weiner LB, Alger KP, Gouveia M, Colelia CB, Schnabel KC, BARKER WH. Influenza surveillance in communitydwelling elderly compared with children. Arch Fam Med 1997; 6: 459-65.

4. Sugaya N, Nerome K, Ishida M, Nerome R, Nagae $M$, TAKeUCHI Y, Osano M. Impact of influenza virus infection as a cause of pediatric hospitalization. J Infect Dis 1992; 165: 373-5.

5. Kappagoda C, Isaacs D, Meuns C, Peat J, De Silva L, O'ConNell A. Critical influenza virus infection. J Paediatr Child Health 2000; 36: 318-21.

6. Glezen WP, Greenberg SB, Atmar RL, Piedra PA, Couch RB. Impact of respiratory virus infections on persons with chronic underlying conditions. JAMA 2000; 283: 499-505.

7. Neuzil KM, Wright PF, Mitchel EF JR, Griffin MR. The burden of influenza illness in children with asthma and other chronic medical conditions. J Pediatr 2000; 137: 856-64.

8. Aymard M, Valette M, Luciani J. Burden of influenza in children: preliminary data from a pilot survey network on community diseases. Pediatr Infect Dis J 2003; 22 (Suppl 10): S211-4.

9. GreENBERG SB. Respiratory viral infections in adults. Curr Opin Pulm Med 2002; 8: 201-8. innecesarias. Aunque esta serie es pequeña en número, ilustra que la infección por VI en niños no es una condición benigna y debe ser considerada para los futuros brotes epidémicos en nuestro medio.

10. Bridges CB, Harper SA, Fukuda K, Uyeki TM, Cox NJ, Singleton JA. Advisory Committee on Immunization Practices. Prevention and control of influenza. Recommendations of the Advisory Committee on Immunization Practices (ACIP). MMWR Recomm Rep 2003; 52: 1-34.

11. Committee on Infectious Diseases. American Academy of Pediatrics. Reduction of the influenza burden in children. Pediatrics 2002; 110: 1246-52.

12. Izurieta HS, Thompson WW, Kramarz P, Shay DK, Davis RL, Destefano F et al. Influenza and the rates of hospitalization for respiratory disease among infants and young children. N Engl J Med 2000; 27: 232-9.

13. Wiшams J, Goodwin P. Influenza immunization in children: good for everyone or reserve for the chosen few? Paediatr Respir Rev 2004; 5: 85-9.

14. Principi N, Espósto S, Marchisio P, Gasparini R, Crovari P. Socioeconomic impact of influenza on healthy children and their families. Pediatr Infect Dis J 2003; 22 (Suppl 10): S207-10.

15. Sотомауов V. Influenza. Boletín de vigilancia en salud pública de Chile. El Vigía 19 2004; 3: 25-28. http://epi.minsal.cl/ (revisado el 02 de octubre de 2004).

16. Centres for Disease Control and Prevention. Department of Health and Human Services. Weekly Report: Influenza Summary update update. http:/ /www.cdc.gov/flu/weekly (revisado el 10 de marzo de 2004).

17. Centres for Disease Control and Prevention. Update: Influenza activity - United State, 2003-04 seasson. MMWR 2004; 53: 284-87. http://www.cdc.gov/ $\mathrm{mmwr} / \mathrm{previev} / \mathrm{mmwrhtml} / \mathrm{mm} 5313 \mathrm{a} 2 . \mathrm{htm}$ (revisado el 11 de mayo de 2004).

18. Sugaya N, Mitamura K, Nirasawa M, Takahashi K. The impact of winter epidemics of influenza and respiratory syncytial virus on paediatric admissions 
to an urban general hospital. J Med Virol 2000; 60: 102-6.

19. NeuZIL KM, Zhu Y, GRIFFin MR, EdwaRds KM, THOMPSON JM, TOLeFson SJ ET AL. Burden of interpandemic influenza in children younger than 5 years: a 25-year prospective study. J Infect Dis 2002; 185: 147-52.

20. Kramarz P, Destefano F, Gargiulo PM, Davis RL, CHen RT, MuLooly JP et AL. Influenza vaccination in children with asthma in health maintenance organizations. Vaccine Safety Datalink Team. Vaccine 2000; 18: 2288-94.

21. Poehling KA, Speroff T, Dittus RS, Griffin MR, Hickson GB, Edwards KM. Predictors of influenza virus vaccination status in hospitalized children. Pediatrics 2001; 108: E99.

22. O'Brien MA, Uyeki TM, Shay DK, Thompson WW, Kleinman K, McAdam A et al. Incidence of outpatient visits and hospitalizations related to influenza in infants and young children. Pediatrics 2004; 113: 585-93.

23. Delpiano L, GuiLén B, Casado MC. Comportamiento clínico-epidemiológico de la influenza en niños hospitalizados. Rev Chil Infect 2003; 20: 159-65.

24. Cox NJ, Subbarao K. Influenza. Lancet 1999; 354: 1277-82.

25. Noyola DE, Demmer GJ. Effect of rapid diagnosis on management of influenza A infections. Pediatr Infect Dis J 2000; 19: 303-7.
26. Belshe RB, Mendelman PM, Treanor J, King J, Gruber WC, Piedra P et al. The efficacy of live attenuated, cold-adapted, trivalent, intranasal influenza virus vaccine in children. $\mathrm{N}$ Engl J Med 1998; 338: 1405-12.

27. Heikinen T, Thint M, Chonmaitree T. Prevalence of various respiratory viruses in the middle ear during acute otitis media. NEngl J Med 1999; 340: 260-4.

28. Juven T, Mertsola J, Waris M, Leinonen M, Meurman O, RoIvainen M ET aL. Etiology of communityacquired pneumonia in 254 hospitalized children. Pediatr Infect Dis J 2000; 19: 293-8.

29. O’Brien KL, Walters MI, Sellman J, Quinlisk P, Regnery H, Schwartz B et al. Severe pneumococcal pneumonia in previously healthy children: the role of preceding influenza infection. Clin Infect Dis 2000; 30: 784-9.

30. Iwane MK, Edwards KM, Szilagyi PG, Walker FJ, GRIFFIN MR, WeINBERG GA et al. New Vaccine Surveillance Network. Population-based surveillance for hospitalizations associated with respiratory syncytial virus, influenza virus, and parainfluenza viruses among young children. Pediatrics 2004; 113: 1758-64.

31. Luce BR, Zangwill KM, Palmer CS, Mendelman PM, YAN L, WolfF MC ET AL. Cost-effectiveness analysis of an intranasal influenza vaccine for the prevention of influenza in healthy children. Pediatrics 2001; 108: E24.

Agradecimientos

A la Señora Teresa Azócar, por su trabajo en el laboratorio de virología. 